\title{
NUMERICAL SOLVABILITY OF A CLASS OF \\ VOLTERRA-HAMMERSTEIN INTEGRAL EQUATIONS WITH NONCOMPACT KERNELS
}

\author{
M. HADIZADEH AND M. MOHAMADSOHI
}

Received 9 October 2003 and in revised form 29 August 2004

We study the numerical solvability of a class of nonlinear weakly singular integral equations of Volterra-Hammerstein type with noncompact kernels. We obtain existence and uniqueness results and analyze the product integration methods for these equations under some verifiable conditions on the kernels and nonlinear functions. The convergence analysis is investigated and finally numerical experiments are given, which confirm our theoretical results.

\section{Introduction}

We consider the nonlinear Volterra-Hammerstein integral equation

$$
y(t)=f(t)+\int_{0}^{t} k\left(\frac{t}{s}\right) g(s, y(s)) \frac{1}{s} d s, \quad t \in[0, T],
$$

where $g$ and $f$ are smooth given functions and the kernel $k$ is a weakly singular kernel.

A convenient setting for the analysis of $(1.1)$ is the space $C^{m}[0, T]$, the Banach space of $m$-times continuously differentiable real-valued functions with uniform norm

$$
\|u\|_{m, \infty}=\max _{0 \leq j \leq m} \max _{0 \leq t \leq T}\left|u^{(j)}(t)\right|
$$

The integral equation (1.1) in the operator form may be written as

$$
y(t)=T G(y(t)), \quad y \in C^{m}[0, T]
$$

where $y(t)$ is the unknown solution and the operator $T$ is defined by

$$
T(w)(t):=f(t)+(K w)(t), \quad t \in[0, T], w \in C^{m}[0, T]
$$


where $K, G$ are the linear integral operator and "substitution" bounded operator, respectively, defined as follows:

$$
\begin{gathered}
(K w)(t):=\int_{0}^{t} k\left(\frac{t}{s}\right) w(s) \frac{1}{s} d s, \quad t \in[0, T], w \in C^{m}[0, T] \\
G(x)(t):=g(t, x(t)), \quad t \in[0, T], x \in C^{m}[0, T] .
\end{gathered}
$$

The difficulty is that the kernel $k$ is not compact, and hence the classical arguments in the convergence analysis of discretization methods for weakly singular equation (1.1) are not applicable. Nonlinear Volterra integral equations of the second kind often occur in Hammerstein form, where the kernel may be weakly singular or otherwise badly behaved. (For obvious source of such equation, see $[2,6,7]$.) Other works that consider problem (1.1) under various assumptions of $k, g$ are $[5,10,13]$.

Equations of type (1.1) can arise in connection with some heat conduction problems with mixed-type boundary conditions and certain diffusion problems. Detailed descriptions and analysis of these models may be found in $[1,10,11,12,14]$.

Actually, few theoretical and numerical methods for some classes of (1.1) are known. Bartoshevich [1], after the application of Watson's transforms and the use of the convolution theorem $[8,13]$, considered the following integral equation:

$$
F(t)+\frac{1}{\sqrt{\pi}} \int_{t}^{\infty} \frac{1}{\sqrt{\ln (s / t)}} \frac{1}{s} F(s) d s=H(t), \quad t>0,
$$

where the function $F(t)$ is the unknown function and $H(t)$ is a given function. The analytical study of this and other related equations has been pursued by several authors $[8,10,12,13]$. In the linear and some particular case of $(1.1)$, it was proved in $[5,13]$ that the equation has a unique solution in the continuity class $C^{m}[0, T]$. For the numerical solution of (1.1), in the linear case, certain classes of product integration methods were studied. Owing to the fact that the kernel $k$ is unbounded in (1.1), the classical arguments of the convergence analysis of the discretization method for weakly singular equations are not applicable. Diogo et al. [3] transformed integral equation of the form (1.6) into the linear form of Volterra integral equation of (1.1) with special noncompact kernel

$$
k(\sigma)=\frac{1}{\sqrt{\pi} \sqrt{\ln \sigma} \sigma^{\mu}}, \quad \mu>1 .
$$

In [13], approximations to the solution of (1.6) of orders one and two were obtained with the Euler and trapezoidal methods, respectively. In [3], a fourth-order Hermite-type collocation method was applied to (1.1) and the analysis and construction of higher-order collocation method has been investigated in [4]. Lima and Diogo in [9] were concerned with the use of a low-order method in conjunction with extrapolation procedures. The results of Tang et al. in [13] had been extended to linear form of Volterra integral equation of (1) in [5]. Recently, Lima and Diogo in [10] have been concerned with the numerical solution of a class of (1.1) with certain values of a real parameter $\mu$ and extrapolation algorithms. They have shown that, although for $0<\mu \leq 1$, the equation possesses an infinite set of solutions, the Euler method converges to a particular solution. (For details, see [10].) 
In the present paper, we further develop the work carried out in [5]. We discuss existence and uniqueness of a solution of (1.1) under some verifiable conditions on the functions $f$ and $g$. We then proceed to investigate the numerical solvability of (1.1), by some classes of product integration methods. Euler-type and product trapezoidal discretization schemes and convergence analysis are considered. Finally, some numerical results are presented in the final section, which support the theoretical results obtained in this paper.

\section{Existence and uniqueness results}

For $T>0$ and $m$ a nonnegative integer, let $V_{m}[0, T]$ denote the normed space of the realvalued function $f$, such that $f \in C^{m}[0, T]$ with

$$
\|f\|_{m, \infty}:=\max _{0 \leq j \leq m} \max _{0 \leq t \leq T}\left|f^{(j)}(t)\right| .
$$

The following result is concerned with existence and uniqueness of the solution of (1.1).

Theorem 2.1. Consider the nonlinear weakly singular integral equation of VolterraHammerstein type (1.1) and make the following assumptions.

(A1) $f(t) \in V_{m}$.

(A2) The kernel $k$ satisfies

$$
\alpha=\int_{1}^{\infty} \frac{|k(\sigma)|}{\sigma} d \sigma<1
$$

(A3) The nonlinear function $g(t, v)$ is defined and continuous on $[0, T] \times \mathbb{R}$.

(A4) The partial derivative $g_{v}^{m}(t, v)=\left(\partial^{m} g / \partial v^{m}\right)(t, v)$ exists and continuous on $[0, T] \times$ $\mathbb{R}$.

(A5) The function $g$ satisfies the Lipshitz condition

$$
\left\|g\left(t, v_{1}\right)-g\left(t, v_{2}\right)\right\|_{m, \infty} \leq L\left\|v_{1}-v_{2}\right\|_{m, \infty}
$$

with constant $L$, where $L \alpha<1$.

Under these assumptions, (1.1) possesses a unique solution $y(t) \in V_{m}$.

Proof. Set $s=\lambda t$, we have

$$
\int_{0}^{t} k\left(\frac{t}{s}\right) g(s, v(s)) \frac{1}{s} d s=\int_{0}^{1} k\left(\frac{1}{\lambda}\right) g(\lambda t, v(\lambda t)) \frac{1}{\lambda} d \lambda .
$$

We define the linear integral operator $K$ for some given kernel function $k$ as follows:

$$
(K w)(t):=\int_{0}^{1} k\left(\frac{1}{\lambda}\right) \frac{1}{\lambda} w(\lambda t) d \lambda, \quad t \in[0, T], w \in V_{m},
$$

and the operator $T$, differing from $K$, merely by a nonhomogeneous term as follows:

$$
T(w)(t):=f(t)+(K w)(t), \quad t \in[0, T], w \in V_{m},
$$


174 Numerical solvability of a class of integral equations

and a continuous bounded operator $G$ as follows:

$$
G(v)(t):=g(t, v(t)), \quad t \in[0, T], v \in V_{m} .
$$

Let $v(t) \in V_{m}$ be an arbitrary function. With the above notation, we define

$$
u=T G(v), \quad v \in C^{m}[0, T] .
$$

Assumptions (A3) and (A4) ensure that $G$ is continuously Fréchet differentiable on $V_{m}$. Since the operator $K$ is linear, then we have

$$
(K G)^{(j)}(v)=K G^{(j)}(v), \quad v \in V_{m} .
$$

If, in addition, (A1) holds, then the $j$ th Fréchet derivative of the operator $T G$ at $t \in[0, T]$ is given by

$$
\left[(T G)^{(j)}(v)\right](t)=K g^{j}(t, v(t))+f^{(j)}(t), \quad t \in[0, T], j=0,1, \ldots, m .
$$

The final assumption (A5) implies that for all $v_{1}, v_{2} \in V_{m}$, where $u_{1}=T G\left(v_{1}\right)$ and $u_{2}=$ $T G\left(v_{2}\right)$, the following inequality holds:

$$
\begin{aligned}
\left|u_{1}^{(j)}-u_{2}^{(j)}\right| & =\left|(T G)^{(j)}\left(v_{1}\right)-(T G)^{(j)}\left(v_{2}\right)\right| \\
& \leq \int_{0}^{1}\left|k\left(\frac{1}{\lambda}\right)\right| \lambda^{j-1}\left|g^{j}\left(t, v_{1}(t)\right)-g^{j}\left(t, v_{2}(t)\right)\right| d \lambda \\
& \leq \int_{0}^{1}\left|k\left(\frac{1}{\lambda}\right)\right| \frac{1}{\lambda} d \lambda|| g\left(t, v_{1}\right)-g\left(t, v_{2}\right) \|_{m, \infty} \\
& \leq L \int_{0}^{1}\left|k\left(\frac{1}{\lambda}\right)\right| \frac{1}{\lambda} d \lambda\left\|v_{1}-v_{2}\right\|_{m, \infty} \quad(j=0,1, \ldots, m) .
\end{aligned}
$$

Noting that, according to (A2), the coefficient of the last term in (2.11) equals $\alpha$, it follows that

$$
\left\|u_{1}-u_{2}\right\|_{m, \infty} \leq L \alpha\left\|v_{1}-v_{2}\right\|_{m, \infty} .
$$

Inequality (2.12) and assumption (A5) imply that the operator TG defines a contraction mapping. Since $V_{m}$ is a complete normed space, $T G$ has a unique fixed point $y \in V_{m}$, such that $y=T G(y)$. This completes the proof of Theorem 2.1.

\section{Numerical solvability and convergence analysis}

In this section, in order to approximate the solution of (1.1), we consider the Euler and product trapezoidal methods and their convergence analysis.

First of all, let $\Pi_{N}: 0=t_{0}<t_{1}<\cdots<t_{N}=T$ be a partition for $[0, T]$ and set $h_{n}=$ $t_{n+1}-t_{n}, h:=\max \left\{h_{n}: 0 \leq n \leq N-1\right\}, I_{n}=\left[t_{n}, t_{n+1}\right)(n=0,1, \ldots, N-1)$.

For given integers $p$ and $d$, with $p>d \geq 0, S_{p-1}^{(d-1)}\left(\Pi_{N}\right) \subset C^{d-1}[0, T]$ will denote the space of piecewise polynomial functions of degree $p-1$, whose knots are the mesh points $\left\{t_{n}: 1 \leq n \leq N-1\right\}$. If $d=0$, there is no continuity requirements at the knots. Note that 
the dimension of this space is given by $\operatorname{dim} S_{p-1}^{(-1)}\left(\Pi_{N}\right)=N p$. If $d=1, S_{p-1}^{(0)}\left(\Pi_{N}\right)$ denotes the space of continuous piecewise polynomials functions of degree $p-1$, whose dimension is equal to $N(p-1)+1$.

In this paper, we will consider only the cases $d=0$ and $d=1$, namely the spaces whose elements are piecewise constant functions and continuous linear polynomials, respectively:

$$
\begin{gathered}
S_{0}^{(-1)}\left(\Pi_{N}\right)=\left\{u(t): u(t)=u_{n}, t \in I_{n}, n=0,1, \ldots, N-1, u(T)=u_{N}\right\}, \\
S_{1}^{(0)}\left(\Pi_{N}\right)=\left\{u(t): u(t)=l_{1, n}(t) u_{n}+l_{2, n}(t) u_{n+1}, t \in\left[t_{n}, t_{n+1}\right], n=0,1, \ldots, N-1\right\},
\end{gathered}
$$

where

$$
l_{1, n}=\frac{t_{n+1}-t}{h_{n}}, \quad l_{2, n}=\frac{t-t_{n}}{h_{n}}
$$

and $u_{j}=u\left(t_{j}\right), j=0,1, \ldots, N$, are constants.

We seek the approximate solution $u$ of $(1.1)$, which belongs to either $S_{0}^{(-1)}\left(\Pi_{N}\right)$ or $S_{1}^{(0)}\left(\Pi_{N}\right)$, which satisfies

$$
u\left(t_{n}\right)=\int_{0}^{t_{n}} k\left(\frac{t_{n}}{s}\right) g(s, u(s)) \frac{1}{s} d s+f\left(t_{n}\right), \quad n=1,2, \ldots, N
$$

with $u(0)=y(0)$, and

$$
u(0)=f(0)+\tilde{\alpha} g(0, u(0))
$$

where

$$
\tilde{\alpha}=\int_{1}^{\infty} \frac{k(\sigma)}{\sigma} d \sigma
$$

Note that (3.4) is a nonlinear equation for the unknown $u(0)$. Since $\left|\tilde{\alpha} g_{v}(0, u(0))\right| \leq \alpha L<$ 1 , then relation (3.4) possesses a unique solution.

Also, noting that

$$
\begin{aligned}
\lim _{t \rightarrow 0^{+}} \int_{0}^{t} k\left(\frac{t}{s}\right) g(s, y(s)) \frac{1}{s} d s & =\lim _{t \rightarrow 0^{+}} \int_{0}^{1} k\left(\frac{1}{\sigma}\right) g(t \sigma, y(t \sigma)) \frac{1}{\sigma} d \sigma \\
& =g(0, y(0)) \int_{0}^{1} k\left(\frac{1}{\sigma}\right) \frac{1}{\sigma} d \sigma \\
& =g(0, y(0)) \int_{1}^{\infty} \frac{k(\sigma)}{\sigma} d \sigma=\tilde{\alpha} g(0, y(0)),
\end{aligned}
$$

we obtain (3.4) by letting $t \rightarrow 0^{+}$in (1.1).

Now, we consider two cases for the numerical solution of (1.1). Case 1. $u(t) \in S_{0}^{(-1)}\left(\Pi_{N}\right)$.

In this case, the following theorem gives the convergence properties of the Euler method. 
176 Numerical solvability of a class of integral equations

Theorem 3.1. Let the function $g(t, y) \in[0, T] \times \mathbb{R}$ in (1.1) satisfy the Lipshitz condition with constant $L$, where $\alpha<1$ and $L<1 / \alpha$. If the partial derivative $g_{y}(t, y):=\partial g(t, y) / \partial y$ exists and is continuous on $[0, T] \times \mathbb{R}$ and the function $f(t)$ belongs to $C^{1}[0, T]$, then (3.3) possesses a unique solution $u(t) \in S_{0}^{(-1)}\left(\Pi_{N}\right)$, moreover, the error function $e(t):=y(t)-$ $u(t)$ satisfies

$$
\|e\|_{\infty}=\max _{0 \leq t \leq T}|e(t)|=O(h) .
$$

Proof. For any function $u \in S_{0}^{(-1)}\left(\Pi_{N}\right),(3.3)$ can be written as

$$
u\left(t_{n}\right)=\sum_{i=0}^{n-1} \int_{t_{i}}^{t_{i+1}} k\left(\frac{t_{n}}{s}\right) \frac{1}{s} d s g\left(t_{i}, u\left(t_{i}\right)\right)+f\left(t_{n}\right), \quad 1 \leq n \leq N .
$$

Since $u(0)$ is given, the above recurrence relation yields a unique solution $u \in S_{0}^{(-1)}\left(\Pi_{N}\right)$. Set $t=t_{n}$ in (1.1) and subtract (3.8) from the resulting equation. Setting $e_{n}(t):=y(t)-$ $u_{n}(t), t \in\left[t_{n}, t_{n+1}\right]$, we obtain

$$
e_{n}\left(t_{n}\right)=\sum_{i=0}^{n-1} \int_{t_{i}}^{t_{i+1}} k\left(\frac{t_{n}}{s}\right) \frac{1}{s}\left[g(s, y(s))-g\left(s, u_{i}(s)\right)\right] d s, \quad 1 \leq n \leq N .
$$

Therefore,

$$
\begin{aligned}
\left|e_{n}\left(t_{n}\right)\right| & \leq \sum_{i=0}^{n-1} \int_{t_{i}}^{t_{i+1}}\left|k\left(\frac{t_{n}}{s}\right)\right| \frac{1}{s}\left|g(s, y(s))-g\left(s, u_{i}(s)\right)\right| d s \\
& \leq L \sum_{i=0}^{n-1} \int_{t_{i}}^{t_{i+1}} \frac{\left|k\left(t_{n} / s\right)\right|}{s} d s|| y(s)-u_{i}(s) \|_{\infty} \\
& \leq L \sum_{i=0}^{n-1} \int_{t_{i}}^{t_{i+1}} \frac{\left|k\left(t_{n} / s\right)\right|}{s} d s|| e_{i}(s) \|_{\infty} .
\end{aligned}
$$

Note that

$$
\int_{0}^{t_{n}}\left|k\left(\frac{t_{n}}{s}\right)\right| \frac{1}{s} d s=\int_{0}^{1}\left|k\left(\frac{1}{\lambda}\right)\right| \frac{1}{\lambda} d \lambda=\int_{1}^{\infty} \frac{|k(\sigma)|}{\sigma} d \sigma=\alpha<1 .
$$

Since $f(t) \in C^{1}[0, T]$, and the conditions of Theorem 2.1 are satisfied, then we have $y(t) \in C^{1}[0, T]$. Now, for $s \in\left[t_{i}, t_{i+1}\right]$, we have

$$
\begin{aligned}
e_{i}(s) & =e_{i}\left(t_{i}\right)+\left(s-t_{i}\right) y^{\prime}\left(\xi_{i}\right), \quad \xi_{i} \in\left(t_{i}, s\right) \\
& =e_{i}\left(t_{i}\right)+O\left(h_{i}\right) .
\end{aligned}
$$

From (3.10) and (3.12), we obtain

$$
\left|e_{n}\left(t_{n}\right)\right| \leq L \sum_{i=0}^{n-1} \int_{t_{i}}^{t_{i+1}} \frac{\left|k\left(t_{n} / s\right)\right|}{s} d s\left|e_{i}\left(t_{i}\right)\right|+O(h) .
$$


Let $E=\max _{1 \leq n \leq N}\left|e_{n}\left(t_{n}\right)\right|$, then (3.11) and (3.13) give

$$
\left|e_{n}\left(t_{n}\right)\right| \leq(\alpha L) E+O(h), \quad 1 \leq n \leq N
$$

This inequality implies that

$$
E \leq(\alpha L) E+O(h)
$$

since $\alpha L<1$, then $E=O(h)$ and from (3.12), the desired result of the theorem is obtained.

Case 2. $u(t) \in S_{1}^{(0)}\left(\Pi_{N}\right)$.

The product trapezoidal method is constructed by approximating $y(t)$ by piecewise linear functions. For any function $u \in S_{1}^{(0)}\left(\Pi_{N}\right)$, (3.3) can be written as

$$
u\left(t_{n}\right)=\sum_{i=0}^{n-1} \int_{t_{i}}^{t_{i+1}} k\left(\frac{t_{n}}{s}\right) g(s, u(s)) \frac{1}{s} d s+f\left(t_{n}\right), \quad n=1,2, \ldots, N
$$

or

$$
u\left(t_{n}\right)=f\left(t_{n}\right)+\alpha_{n 1} g(0, u(0))+\sum_{j=1}^{n-1}\left(\alpha_{n, j+1}+\beta_{n, j}\right) g\left(t_{j}, u\left(t_{j}\right)\right)+\beta_{n n} g\left(t_{n}, u\left(t_{n}\right)\right)
$$

where

$$
\begin{aligned}
& \alpha_{n, j+1}=\int_{t_{j}}^{t_{j+1}} l_{1, j}(s) k\left(\frac{t_{n}}{s}\right) \frac{1}{s} d s, \\
& \beta_{n, j+1}=\int_{t_{j}}^{t_{j+1}} l_{2, j}(s) k\left(\frac{t_{n}}{s}\right) \frac{1}{s} d s .
\end{aligned}
$$

Note that (3.17) represents a nonlinear system (equation) for the unknown $u\left(t_{n}\right)$. Since $0 \leq l_{2, n-1}(s) \leq 1$ for $s \in\left[t_{n-1}, t_{n}\right]$, we have

$$
\begin{gathered}
\left|\beta_{n n}\right|=\left|\int_{t_{n-1}}^{t_{n}} l_{2, n-1}(s) k\left(\frac{t_{n}}{s}\right) \frac{1}{s} d s\right| \leq \int_{t_{n-1}}^{t_{n}}\left|k\left(\frac{t_{n}}{s}\right)\right| \frac{1}{s} d s<\int_{0}^{t_{n}}\left|k\left(\frac{t_{n}}{s}\right)\right| \frac{1}{s} d s=\alpha, \\
\left|g_{u}^{\prime}\left(t_{n}, u\left(t_{n}\right)\right)\right| \leq L,
\end{gathered}
$$

then

$$
\left|\beta_{n n} g_{u}^{\prime}\left(t_{n}, u\left(t_{n}\right)\right)\right|<L \alpha<1
$$

and it can be seen that the recurrence relation (3.17) yields a unique solution $u(t) \in$ $S_{1}^{(0)}\left(\Pi_{N}\right)$. 
178 Numerical solvability of a class of integral equations

Theorem 3.2. Let the function $g(t, y) \in[0, T] \times \mathbb{R}$ in (1.1) satisfy the Lipshitz condition with constant $L$, where $\alpha<1$ and $L<1 / \alpha$. If the partial derivative $g_{y}^{2}(t, y):=\partial g_{y}(t, y) / \partial y$ exists and is continuous on $[0, T] \times \mathbb{R}$ and $f(t) \in C^{2}[0, T]$, then (3.3) possesses a unique solution $u(t) \in S_{1}^{(0)}\left(\Pi_{N}\right)$ and the error function $e(t):=y(t)-u(t)$ satisfies

$$
\|e\|_{\infty}=\max _{0 \leq t \leq T}|e(t)|=O\left(h^{2}\right) .
$$

Proof. Following Theorem 3.1, we obtain (3.9). Similarly to what we have done in the previous theorem, if we set $e_{i}(s)=y(s)-u_{i}(s)$, then since $f(t) \in C^{2}[0, T]$ and $L \alpha<1$, we obtain from Theorem 2.1 that $y(t) \in C^{2}[0, T]$.

For $s \in\left[t_{i}, t_{i+1}\right]$, we have

$$
\begin{aligned}
e_{i}(s) & =l_{1, i}(s) y\left(t_{i}\right)+l_{2, i}(s) y\left(t_{i+1}\right)+O\left(h_{i}^{2}\right)-l_{1, i}(s) u\left(t_{i}\right)-l_{2, i}(s) u\left(t_{i+1}\right) \\
& =l_{1, i}(s) e_{i}\left(t_{i}\right)+l_{2, i}(s) e_{i+1}\left(t_{i+1}\right)+O\left(h_{i}^{2}\right) .
\end{aligned}
$$

Noting that for $s \in\left[t_{i}, t_{i+1}\right], l_{1, i}(s) \geq 0, l_{2, i}(s) \geq 0, l_{1, i}(s)+l_{2, i}(s)=1,(3.22)$ gives

$$
\left|e_{i}(s)\right| \leq\left(l_{1, i}(s)+l_{2, i}(s)\right) E+O\left(h^{2}\right)=E+O\left(h^{2}\right),
$$

where $E=\max \left\{\left|e_{n}\left(t_{n}\right)\right|: 1 \leq n \leq N\right\}$.

Applying (3.23) and (3.11) to (3.10), we obtain

$$
\left|e_{n}\left(t_{n}\right)\right| \leq(\alpha L) E+O\left(h^{2}\right)
$$

which implies that

$$
E \leq(\alpha L) E+O\left(h^{2}\right)
$$

Since $\alpha L<1,(3.25)$ yields that $E=O\left(h^{2}\right)$. Then, recalling (3.23), we obtain the result of the theorem.

\section{Numerical experiments}

In order to illustrate the theoretical results of Section 3 and for computational purpose, we consider the following test problems.

Example 4.1. Consider the nonlinear Volterra-Hammerstein integral equation with noncompact kernel

$$
y(t)=t+\frac{1}{t+1}\left(e^{-(t+1)}-1\right)+\int_{0}^{t} \frac{1}{t} e^{-s / t} e^{-y(s)} d s, \quad t \in[0,1]
$$

where $k(\sigma)=(1 / \sigma) e^{-1 / \sigma}$. The exact solution of this equation is $y(t)=t$.

Note that

$$
\int_{1}^{\infty} \frac{k(\sigma)}{\sigma} d \sigma=1-\frac{1}{e}<1
$$


Table 4.1

\begin{tabular}{lcccc}
\hline$h=2^{-k}$ & $E_{N}^{(0)}$ & $\alpha^{(0)}$ & $E_{N}^{(1)}$ & $\alpha^{(1)}$ \\
\hline$k=3$ & $3.1812 E-2$ & 0.97303 & $6.1834 E-4$ & 1.9744 \\
$k=4$ & $1.6206 E-2$ & 0.98641 & $1.5736 E-4$ & 1.9874 \\
$k=5$ & $8.1797 E-3$ & 0.99318 & $3.9684 E-5$ & 1.9938 \\
$k=6$ & $4.1092 E-3$ & 0.99658 & $9.9640 E-6$ & 1.9969 \\
$k=7$ & $2.0595 E-3$ & 0.99829 & $2.4964 E-6$ & 1.9984 \\
$k=8$ & $1.0310 E-3$ & 0.99914 & $6.2476 E-7$ & 1.9992 \\
$k=9$ & $5.1579 E-4$ & 0.99957 & $1.5627 E-7$ & 1.9996 \\
$k=10$ & $2.5797 E-4$ & 0.99979 & $3.9079 E-8$ & 1.9998 \\
$k=11$ & $1.2900 E-4$ & - & $9.7711 E-9$ & - \\
\hline
\end{tabular}

and so the conditions of Theorem 2.1 are satisfied. We choose uniform partitions with $h=1 / N, N=2^{k}, k=3, \ldots, 11$. The solution of this equation will be approximated by the Euler Method in the space $S_{0}^{(-1)}\left(\Pi_{N}\right)$ and by the product trapezoidal scheme in the space $S_{1}^{(0)}\left(\Pi_{N}\right)$, respectively.

The maximum absolute errors and a summary of the predicted convergence orders are given in Table 4.1 .

Example 4.2. In this example, we consider the nonlinear weakly singular VolterraHammerstein integral equation with algebraic nonlinearity

$$
y(t)=-\frac{t^{4}}{10}+\frac{5 t^{2}}{6}+\frac{3}{8}+\int_{0}^{t} \frac{1}{2 t} y^{2}(s) d s, \quad t \in[0,1],
$$

where $k(\sigma)=1 / 2 \sigma$. Here, the conditions of Theorem 2.1 are satisfied and we have

$$
\int_{1}^{\infty} \frac{k(\sigma)}{\sigma} d \sigma=0.5<1
$$

The exact solution of this equation is $y(t)=t^{2}+1 / 2$.

We refrain from going into details. Using the same notations and methods as implemented in the previous example, we give the errors and the associated rates of convergence of the Euler and product trapezoidal methods in Table 4.2.

All computations are carried out with Mathematica programming. For the ease of notation, we define the following in Tables 4.1 and 4.2:

$$
\begin{array}{ll}
E_{N}^{(0)}=\max \left\{\left|y\left(t_{i}\right)-u\left(t_{i}\right)\right|: t_{i} \in[0,1]\right\}, & \alpha^{(0)}=\log _{2}\left(\frac{E_{N}^{(0)}}{E_{2 N}^{(0)}}\right), \\
E_{N}^{(1)}=\max \left\{\left|y\left(t_{i}\right)-v\left(t_{i}\right)\right|: t_{i} \in[0,1]\right\}, & \alpha^{(1)}=\log _{2}\left(\frac{E_{N}^{(1)}}{E_{2 N}^{(1)}}\right),
\end{array}
$$


180 Numerical solvability of a class of integral equations

Table 4.2

\begin{tabular}{lcccc}
\hline$h=2^{-k}$ & $E_{N}^{(0)}$ & $\alpha^{(0)}$ & $E_{N}^{(1)}$ & $\alpha^{(1)}$ \\
\hline$k=3$ & $8.0518 E-2$ & 0.85884 & $7.4244 E-3$ & 1.9824 \\
$k=4$ & $4.4397 E-2$ & 0.92287 & $1.8789 E-3$ & 1.9826 \\
$k=5$ & $2.3418 E-2$ & 0.95933 & $4.7540 E-4$ & 1.9866 \\
$k=6$ & $1.2044 E-2$ & 0.97902 & $1.1996 E-4$ & 1.9902 \\
$k=7$ & $6.1100 E-3$ & 0.98931 & $3.0194 E-5$ & 1.9931 \\
$k=8$ & $3.0777 E-3$ & 0.99460 & $7.5850 E-6$ & 1.9951 \\
$k=9$ & $1.5446 E-3$ & 0.99728 & $1.9027 E-6$ & 1.9965 \\
$k=10$ & $7.7377 E-4$ & 0.99863 & $4.7683 E-7$ & 1.9976 \\
$k=11$ & $3.8725 E-4$ & - & $1.1941 E-7$ & - \\
\hline
\end{tabular}

where $u\left(t_{i}\right)$ and $v\left(t_{i}\right)$ are the approximate solutions of the Euler and product trapezoidal schemes, respectively.

\section{Concluding remarks}

This paper has introduced a class of nonlinear Volterra-Hammerstein integral equations with noncompact kernels. The existence, the uniqueness, and the numerical solution of these equations by some product integration methods have been investigated. Owing to some special properties of the kernel, the classical arguments in the convergence analysis of discretization methods are not applicable. Applying other numerical schemes, for example, collocation methods and obtaining higher-order convergence give rise to some difficulties in error analysis, which we will consider in other works.

\section{Acknowledgments}

The authors express their gratefulness to the referees for their scrupulous corrections and valuable suggestions for improvement of this paper. This research was in part supported by a grant from Institute for Studies in Theoretical Physics and Mathematics (IPM) (no. 82650024).

\section{References}

[1] M. A. Bartoshevich, On a class of Watson transforms, Dokl. Akad. Nauk SSSR 220 (1975), 761764 (Russian).

[2] H. Brunner, Implicitly linear collocation methods for nonlinear Volterra equations, Appl. Numer. Math. 9 (1992), no. 3-5, 235-247.

[3] T. Diogo, S. McKee, and T. Tang, A Hermite-type collocation method for the solution of an integral equation with a certain weakly singular kernel, IMA J. Numer. Anal. 11 (1991), no. 4, 595605.

[4] Collocation methods for second-kind Volterra integral equations with weakly singular kernels, Proc. Roy. Soc. Edinburgh Sect. A 124 (1994), no. 2, 199-210. 
[5] H. Guoqiang, K. Hayami, and W. Jiong, Product integration method for Volterra integral equation with noncompact kernel, Appl. Math. Comput. 121 (2001), no. 2-3, 363-371.

[6] H. Kaneko and Y. Xu, Degenerate kernel method for Hammerstein equations, Math. Comp. 56 (1991), no. 193, 141-148.

[7] S. Kumar, Superconvergence of a collocation-type method for simple turning points of Hammerstein equations, Math. Comp. 50 (1988), no. 182, 385-398.

[8] W. Lamb, A spectral approach to an integral equation, Glasg. Math. J. 26 (1985), no. 1, 83-89.

[9] P. Lima and T. Diogo, An extrapolation method for a Volterra integral equation with weakly singular kernel, Appl. Numer. Math. 24 (1997), no. 2-3, 131-148.

[10] Numerical solution of a nonuniquely solvable Volterra integral equation using extrapolation methods, J. Comput. Appl. Math. 140 (2002), no. 1-2, 537-557.

[11] S. McKee, Volterra integral and integro-differential equations arising from problems in engineering and science, Bull. Inst. Math. Appl. 24 (1988), no. 9-10, 135-138.

[12] P. G. Rooney, On an integral equation on Šub-Sizonenko, Glasg. Math. J. 24 (1983), no. 2, 207210.

[13] T. Tang, S. McKee, and T. Diogo, Product integration methods for an integral equation with logarithmic singular kernel, Appl. Numer. Math. 9 (1992), no. 3-5, 259-266.

[14] J. A. Šub Sizonenko, Inversion of an integral operator by the method of expansion with respect to orthogonal Watson operators, Siberian Math. J. 20 (1979), no. 2, 318-321.

M. Hadizadeh: Department of Mathematics, Faculty of Science, K.N. Toosi University of Technology, P.O. Box 16315-1618, Tehran, Iran; School of Mathematics, Institute for Studies in Theoretical Physics and Mathematics (IPM), P.O. Box 19395-5746, Tehran, Iran

E-mail address: hadizadeh@kntu.ac.ir

M. Mohamadsohi: Department of Mathematics, Faculty of Science, K.N. Toosi University of Technology, P.O. Box 16315-1618, Tehran, Iran

E-mail address: mohamadsohi@kntu.ac.ir 


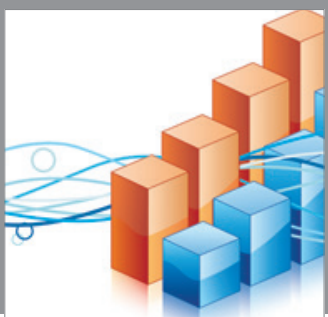

Advances in

Operations Research

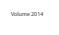

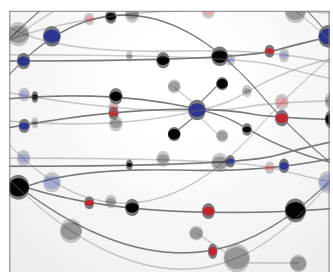

\section{The Scientific} World Journal
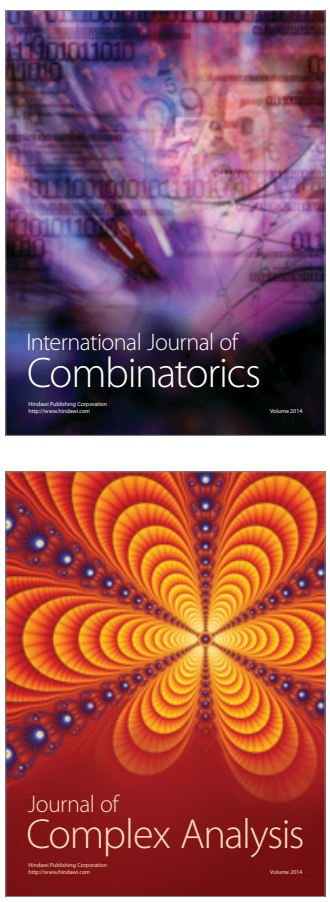

International Journal of

Mathematics and

Mathematical

Sciences
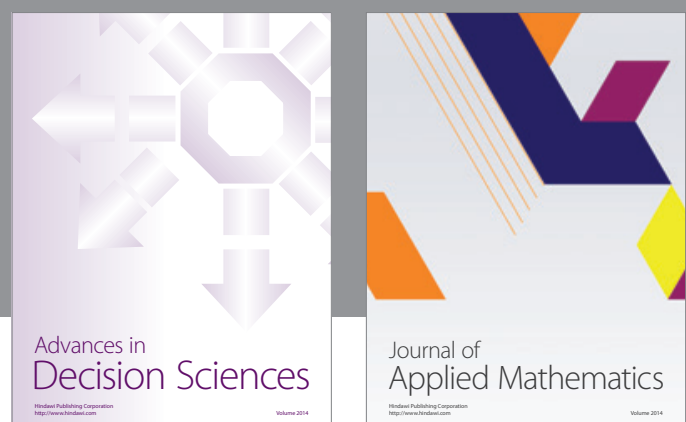

Journal of

Applied Mathematics
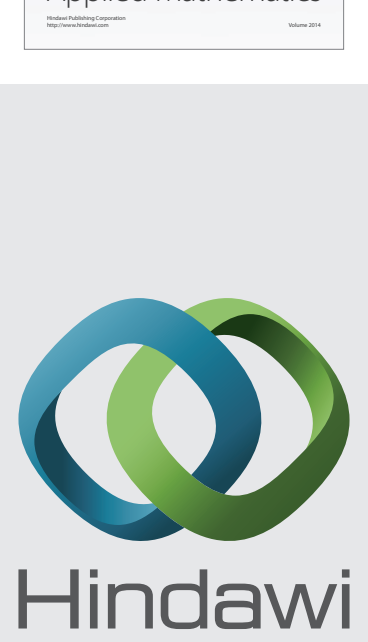

Submit your manuscripts at http://www.hindawi.com
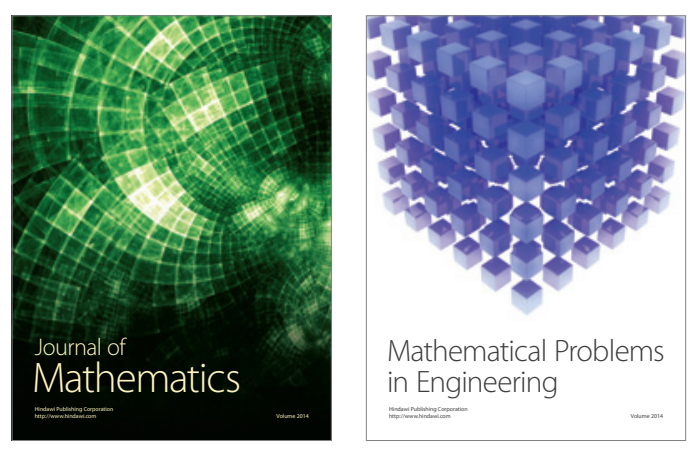

Mathematical Problems in Engineering
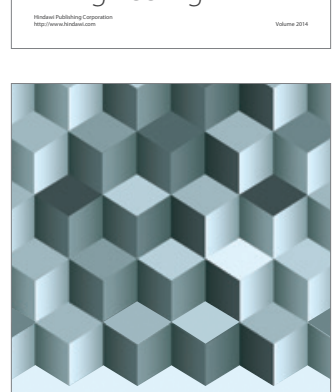

Journal of

Function Spaces
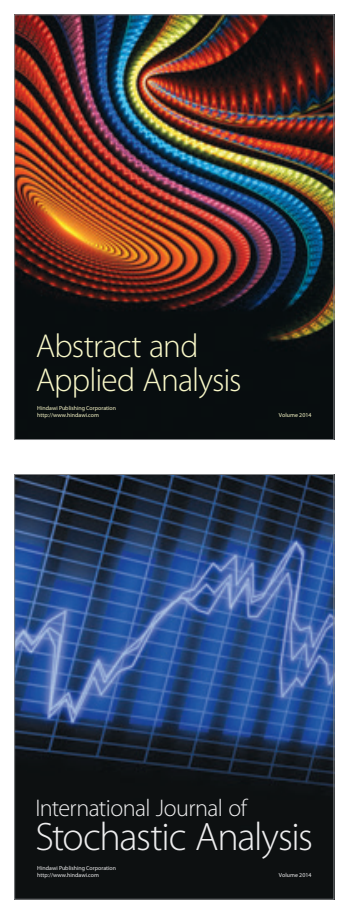

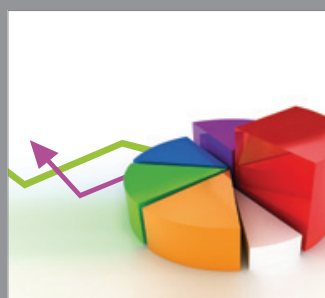

ournal of

Probability and Statistics

Promensencen
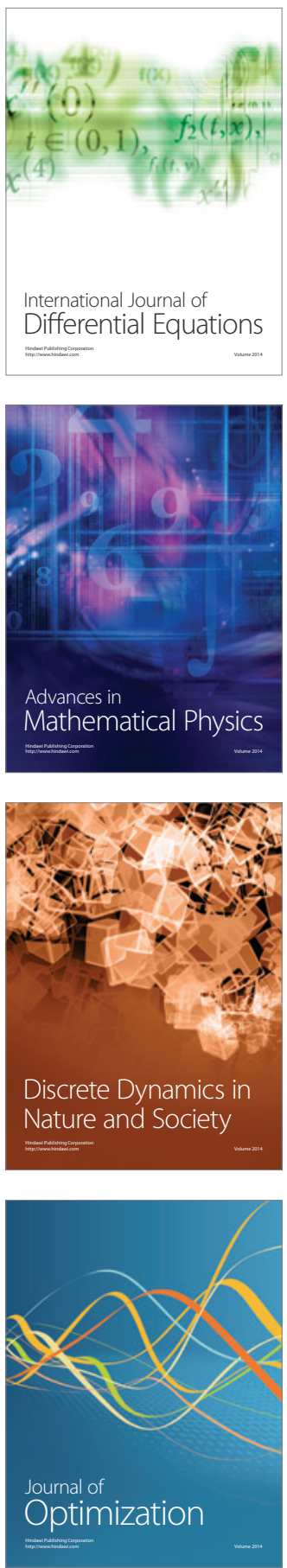